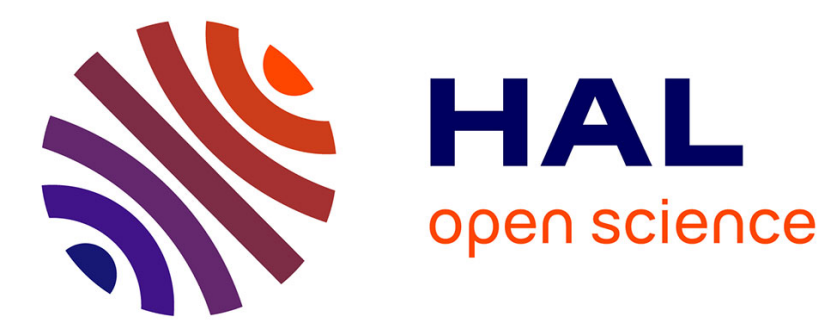

\title{
Time-coherency of Bayesian priors on transient semi-Markov chains for audio-to-score alignment Philippe Cuvillier
}

\section{To cite this version:}

Philippe Cuvillier. Time-coherency of Bayesian priors on transient semi-Markov chains for audio-toscore alignment. MaxEnt 2014, SEE, Sep 2014, Amboise, France. hal-01080235

\section{HAL Id: hal-01080235 \\ https://hal.inria.fr/hal-01080235}

Submitted on 4 Nov 2014

HAL is a multi-disciplinary open access archive for the deposit and dissemination of scientific research documents, whether they are published or not. The documents may come from teaching and research institutions in France or abroad, or from public or private research centers.
L'archive ouverte pluridisciplinaire HAL, est destinée au dépôt et à la diffusion de documents scientifiques de niveau recherche, publiés ou non, émanant des établissements d'enseignement et de recherche français ou étrangers, des laboratoires publics ou privés. 


\title{
Time-coherency of Bayesian priors on transient semi-Markov chains for audio-to-score alignment
}

\author{
Philippe Cuvillier \\ MuTant project-team - Ircam, Inria, UPMC, CNRS \\ Ircam - 1, place Igor-Stravinsky - Paris, France
}

\begin{abstract}
This paper proposes a novel insight to the problem of real-time alignment with Bayesian inference. When a prior knowledge about the duration of events is available, Semi-Markov models allow the setting of individual duration distributions but give no clue about their choice. We propose a criterion of temporal coherency for such applications and show it might be obtained with the right choice of estimation method. Theoretical insights are obtained through the study of the prior state probability of transient semi-Markov chains.
\end{abstract}

Keywords: Bayesian inference, sequential estimation, hidden semi-Markov models, semi-Markov chains

\section{INTRODUCTION}

Many signals are structured as time-contiguous events which generate specific observations, e.g. music, speech or text. In music, basic events may be notes (pitched sounds) and silences. To recognize the sequence of events that generates an observed signal, probabilistic models [1] are relevant when statistical relationships between observation and events are known. In particular, the Hidden Markov Models (HMM) [2] assume that the signal is stationary on time-intervals and identify them with the occupancy of a hidden state. Once the state-space and the statistical priors are specified, Bayesian inference can be readily computed to recognize the state-sequence.

Score alignment [3] is a Music Information Retrieval (MIR) task consisting of synchronizing a musical performance with its score, i.e. the sequence of notes. Since ordering of events is known, recognition boils down to alignment. Among the numerous applications of HMM, music has an outstanding property: a music score assigns to each event its nominal duration, i.e. a prior information on their likely duration.

A crucial and undermined question is about the modeling of nominal duration. This investigation is built on the framework of hidden semi-Markov models (HSMM) as it provides explicit choice of the prior duration model. In section 2 we detail this motivation and briefly introduce HSMM. This generalization of HMM involves many Bayesian priors whose tuning is a major issue. To this aim, most probabilistic models rely on learning with training datasets. This paper presents an alternative based on a theoretical study of prior probability distributions of semi-Markov processes.

In section 3, we state our condition of time-coherency, and explain how the Viterbi estimation does not fulfill it. In section 4, we investigate how the Forward estimation may fulfill it or not depending on several distribution properties of the Bayesian priors. 


\section{BACKGROUND \& MOTIVATION}

\section{Semi-Markov models for alignment}

Hidden semi-Markov models were introduced in [4] as a generalization of HMM. Both are defined with two stochastic processes [2]. The process $\left(S_{t}\right)_{t \in \mathbb{N}^{*}}$ is a discretetime homogeneous Markov chain on a discrete state-space $E \stackrel{\text { def }}{=}\{1,2, \ldots, J\}$, finite or not $(J=\infty)$. Since its realizations $\left(s_{t}\right)_{t \in \mathbb{N}^{*}}$ are not known, they are called hidden states. The observation $\left(o_{t}\right)_{t \in \mathbb{N}^{*}}$, e.g. the audio signal, is considered as a realization of the second process $\left(O_{t}\right)_{t \in \mathbb{N}^{*}}$. We denote $\mathbb{N} \stackrel{\text { def }}{=}\{0,1, \ldots\}, \mathbb{N}^{*} \stackrel{\text { def }}{=}\{1,2, \ldots\}$ and $S_{t}^{t+u} \stackrel{\text { def }}{=}$ $\left(S_{t}, S_{t+1}, \ldots, S_{t+u}\right)$.

In such probabilistic models, the duration spent on a state $j$ is a time-homogeneous random variable. Its law is called the occupancy distribution $d_{j}(u) \stackrel{\text { def }}{=} \mathbb{P}\left(S_{t+u+1} \neq\right.$ $\left.j, S_{t+2}^{t+u}=j \mid S_{t+1}=j, S_{t} \neq j\right)$ for $u \in \mathbb{N}^{*}$. For a Markov state with self-transition $p$, $d_{j}$ would implicitly be a geometric law $d_{j}(u)=(1-p) p^{u-1}$. Assuming that $\left(S_{t}\right)_{t}$ is a semi-Markov chain allows choosing each Bayesian prior $d_{j}$ as any probabilistic mass function (pmf) on $\mathbb{N}^{*}$.

A semi-Markov chain consists of two additional choices per state $j$ : the initial probability $\pi(j) \stackrel{\text { def }}{=} \mathbb{P}\left(S_{1}=j\right)$, and the transition probabilities $p_{i j} \stackrel{\text { def }}{=} \mathbb{P}\left(S_{t+1}=j \mid S_{t+1} \neq i, S_{t}=\right.$ i) with $p_{i i}=0$. In alignment tasks, left-to-right topologies of transition probabilities conveniently model the prior information of ordering. This study exclusively deals with the simplest topology, the linear semi-Markov chains: $\forall i, j, \quad p_{i j}=\delta_{i, i+1}$ and $\pi_{j}=\delta_{1, j}$ (see figure 3 for an example).

Moreover, the hidden model paradigm describes how states $\left(S_{t}\right)$ influence observations $\left(O_{t}\right)$ using observation probabilities $b_{j}\left(o_{t}^{t+u}\right) \stackrel{\text { def }}{=} \mathbb{P}\left(O_{t}^{t+u}=o_{t}^{t+u} \mid S_{t}^{t+u}=j\right)$.

\section{Modeling prior information of duration with HSMMs}

Inference with semi-Markov models requires a careful design of the prior distributions $d_{j}$ for each state. Usual approaches rely on statistical learning. The Baum-Welch algorithm, i.e. the HMM version of Expectation-Maximization (EM), has been adapted to semi-Markov models [5]. But this non-parametric algorithm requires huge training datasets. Consequently, most implementations prefer a parametric EM [6] to learn the occupancy distributions over a parametric family of probabilities, e.g. Gamma, Poisson, log-normal, Negative Binomial laws.

This study aims at elaborating a criterion so as to justify or disqualify such choices. It is built on an interesting property of our application: musical events are associated with a reference duration. Indeed a music score provides the prior tempo and prior durations for all notes.

We denote this quantity the nominal duration $l_{j}$. Although a few music alignment systems like [7] willingly discard this prior information, this work considers duration as an explicit element of modeling and makes the following assumption: two events 
with identical nominal duration should get identical occupancy distributions. So the duration model consists of a set of durations $L \subset \mathbb{R}_{+}$and a duration-indexed family of pmfs $\left(d_{l}\right)_{l \in L}$ such that for all state $j, l_{j} \in L$ and $d_{j}=d_{l_{j}}$. This framework sharpens the problematic: are there coherent mappings from nominal durations $l$ to distributions $d_{l}$ ?

\section{CRITERION OF COHERENCY FOR PRIORS OF DURATION}

\section{Hypothesis of non-discriminative observation}

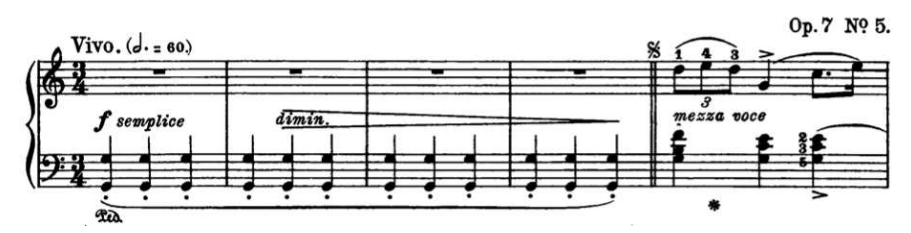

FIGURE 1. Music score of the Mazurka Op. 7 No. 5 by F. Chopin. It begins with a long sequence of repeated events, i.e. states with identical observation probabilities.

Our definition of time-coherency emerges from the following fact: music scores might be composed of very long sequences of "repeated events" such that the one in figure 1 . What would happen if all states $j \in E$ share the same observation probabilities? We call non-discriminative observation such a model where $b_{1}=b_{2}=\ldots \stackrel{\text { def }}{=} b$. Note that this assumption may model other realistic situations of Bayesian inference such as missing observations [8].

\section{Ideal behavior with non-discriminative observation}

We state our criterion of time-coherency. Its rationale is simple: if the observation probabilities do not discriminate states, then the inference should respect the states ordering and their nominal durations as these are the only available information.

Time-coherency criterion 1. On a linear chain with non-discriminative observation, the inference successively decodes states $1,2,3, \ldots$ at time steps $1,1+l_{1}, 1+l_{1}+l_{2}, \ldots$ and assigns to each state $j$ a duration which is equal to its nominal duration $l_{j}$.

The hypothesis of non-discriminative observations makes the posterior probabilities equal to the prior probabilities:

$$
\forall t \in \mathbb{N}^{*}, \quad \mathbb{P}\left(S_{1}, \ldots, S_{t} \mid O_{1}, \ldots, O_{t}\right)=\mathbb{P}\left(S_{1}, \ldots, S_{t}\right) .
$$

Indeed, the Markovian assumption implies that $\mathbb{P}\left(O_{1}^{t} \mid S_{1}^{t}\right)=\prod_{u=1}^{t} \mathbb{P}\left(O_{u} \mid S_{u}\right)=$ $\prod_{u=1}^{t} b_{S_{u}}\left(O_{u}\right)$. Assuming that $b_{S_{u}}=b$ gives $\mathbb{P}\left(O_{1}^{t} \mid S_{1}^{t}\right)=\prod_{u=1}^{t} b\left(O_{u}\right)=\mathbb{P}\left(O_{1}^{t}\right)$, so $\left(S_{t}\right)$ and $\left(O_{t}\right)$ are independent. Thus, the inferred quantities become independent of the observations. Whether the criterion is fulfilled only depends on the underlying semi-Markov chain $\left(S_{t}\right)_{t}$ and the estimation method. 
Offline alignments estimate the most likely sequence $s_{1}^{T}$ at final time $T$, using the so-called Viterbi algorithm. But online alignments make sequential estimations. At each time $t=1 \ldots T$, they could either estimate the partial sequence $s_{1}^{t}$ or the most likely current state $\hat{s}_{t}$. The Viterbi alignment is defined as $\hat{s}_{t}$ such that $\hat{s}_{1}^{t} \stackrel{\text { def }}{=} \arg \max _{s_{1}, \ldots, s_{t} \in E^{t}} \mathbb{P}\left(S_{1}^{t}=\right.$ $\left.s_{1}^{t} \mid O_{1}^{t}=o_{1}^{t}\right)$. The Forward alignment is defined as $\hat{s}_{t}=\arg \max _{s_{t} \in E} \mathbb{P}\left(S_{t}=s_{t} \mid O_{1}^{t}=o_{1}^{t}\right)$. These quantities are obtained using the recursive equations detailed in [5].

\section{Failure of the Viterbi estimation}

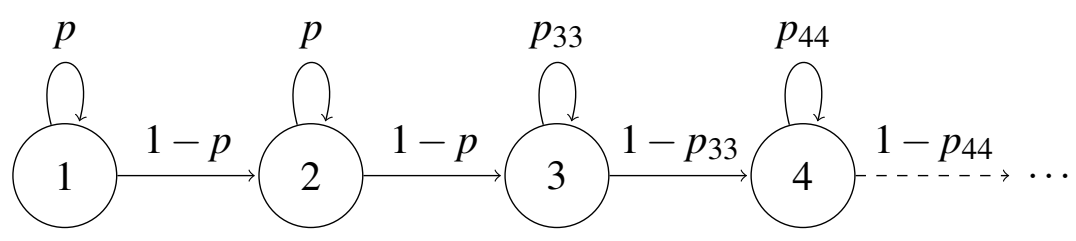

FIGURE 2. Example of a linear Markov chain with identical first two states: $p_{11}=p_{22}=p$.

Our first claim is that the Viterbi alignment fails to be time-coherent. Let us illustrate it with the example in figure 2: a linear Markov chain with identical first two states.

Let $\mathbf{S}=\left(S_{1}, \ldots, S_{t+1}\right)$ be an admissible (i.e. non-decreasing) path. If $\mathbf{S}$ ends at $S_{t+1}=$ 1 , then $\mathbb{P}(\mathbf{S})=p^{t}$. If $S_{t+1}=2$, then $\mathbb{P}(\mathbf{S})=(1-p) p^{t-1}$. So, if $p>1 / 2$ then state 1 is more likely than state 2 at all times for the Viterbi estimation, whereas if $p<1 / 2$ state 2 is more likely than state 1 at all times $t>1$.

This simplistic example could be extended to semi-Markov chains for a wide class of occupancy distributions, but it is enough to reveal the lack of coherency of Viterbi alignments: the parameters cannot be tuned to take into account the nominal duration $l_{1}$.

\section{COHERENCY OF THE FORWARD ESTIMATION}

Our second claim is that the Forward alignment may be time-coherent. This section introduces sufficient conditions on occupancy distributions that imply criterion 1.

Recall that under non-discriminative observation, $F_{j}(t)=\mathbb{P}\left(S_{t}=j\right)$. Let $\mathbf{F}(t) \stackrel{\text { def }}{=}\left(F_{1}(t), F_{2}(t), \ldots\right)$ denote the state probability distribution on $E$, and $\mathbf{M}\left[\mathbf{F}_{t}\right] \stackrel{\text { def }}{=} \arg \max _{j \in E} F_{j}(t)$ denote its mode.

Criterion 1 has the following translation:

$$
\forall t \in \mathbb{N}^{*}, \quad \mathbf{M}\left[\mathbf{F}_{t}\right]=j \Leftrightarrow 1 \leq t-\left(l_{1}+\ldots+l_{j}\right) \leq l_{j+1}
$$

On linear chains, the prior state probabilities are given by successive convolutions. Using the recursive equations detailed in [5, Section 3.2], a simple induction over states $j$ proves that

$$
F_{j}(t)=\frac{1}{K(t)} \cdot \begin{cases}d_{1} * d_{2} * \ldots * d_{j-1} * D_{j}(t) & \text { if } j>1 \\ D_{1}(t) & \text { else }\end{cases}
$$




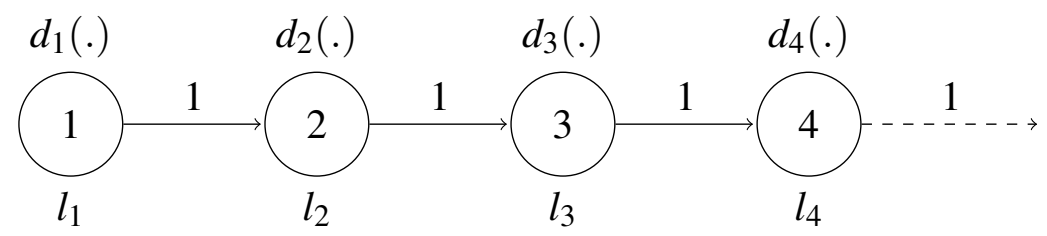

FIGURE 3. Example of a linear semi-Markov chain.

where $D_{j}(t)=\sum_{u \geq t} d_{j}(u)$ is the survivor distribution associated to $d_{j}$, and $K(t)=$ $D_{1}(t)+d_{1} * D_{2}(t)+d_{1} * d_{2} * D_{3}(t)+\ldots$ is an unimportant normalization constant.

\section{The case of first two states}

Since state 1 is the most likely one at first time step $t=1$, we begin with a comparison between states 1 and 2 by studying the evolution of the probability ratio $\frac{F_{1}(t)}{F_{2}(t)}$. The Forward estimation respects the criterion 1 for these two states if and only if

$$
\forall j \in E, \quad \frac{F_{2}(t)}{F_{1}(t)}\left\{\begin{array}{ll}
\leq 1 & \text { if } t \leq l_{1} \\
>1 & \text { if } t>l_{1}
\end{array} .\right.
$$

Proposition 1. Let us denote $\mathbf{m}\left[d_{1}\right] \stackrel{\text { def }}{=} \max \left\{t \mid D_{1}(t) \geq 1 / 2\right\}$ the median of $d_{1}$. Then for any distribution $d_{2}$,

$$
t \leq \mathbf{m}\left[d_{1}\right] \quad \Rightarrow \quad F_{1}(t) \geq F_{2}(t) .
$$

Reciprocally there exists a distribution $d_{2}$ such that $t>\mathbf{m}\left[d_{1}\right] \Rightarrow F_{1}(t)<F_{2}(t)$.

Proof. Since $D_{2}(t) \leq 1$ for all $t, \sum_{u=1}^{t-1} d_{1}(u) D_{2}(t-u) \leq \sum_{u=1}^{t-1} d_{1}(u)$ so $F_{2}(t) \leq 1-D_{1}(t)$ and $F_{2}(t)-F_{1}(t) \leq 1-2 D_{1}(t)$. Since $D_{1}$ is non-increasing, if $t \leq \mathbf{m}\left[d_{1}\right]$ then $D_{1}(t) \geq 1 / 2$ and $1-2 D_{1}(t) \leq 0$.

For the necessary condition one may consider the trivial distribution $d_{2}(t)=\delta_{\mathbf{m}\left[d_{1}\right]}(t)$.

Last proposition tells that the median of $d_{1}$ is a lower bound for the duration assigned to state 1 . Thus it prescribes choosing every distribution $d_{l}$ such that its median is $\lfloor l\rfloor$. But even if this result provides the first half of the criterion 1, the other half may not be fulfilled in the general case.

\section{Uncoherency of heavy-tailed distributions}

First, we give a negative example of distributions that never fulfill the criterion. An important feature of probability distributions is their asymptotic speed of decay. This 
feature is related to the radius of convergence $R_{d} \in[1,+\infty]$ of the probability generating function of $d$, denoted $Z[d](z) \stackrel{\text { def }}{=} \sum_{n \in \mathbb{N}} d(n) z^{n}$.

Definition 1. A discrete distribution $d$ is said to be heavy-tailed if $R_{d}=1$, and lighttailed if not.

Convolutions of heavy-tailed distributions have been thoroughly studied. We borrow the following non-trivial result from [9].

Proposition 2. If $d_{1}$ is an heavy-tailed pmf, then

$$
\liminf _{t \rightarrow \infty} \frac{\sum_{u=0}^{t-1} d_{1}(u) D_{1}(t-u)}{D_{1}(t)}=1
$$

If the two states have the same heavy-tailed occupancy distribution, then state 1 is decoded an infinite number of time. So, the criterion is never fulfilled. This fact discards using such pmfs as Bayesian priors.

\section{Coherency of IHR distributions}

Nevertheless, using light-tailed distributions does not guarantee neither the criterion. But another notion of tail analysis helps checking whether the criterion hold or not.

Definition 2. A distribution $d$ is Increasing Hazard Rate (IHR) if its hazard rate $h(n) \stackrel{\text { def }}{=}$ $\frac{d(n)}{D(n)}$ is non-decreasing.

Proposition 3. Let $d_{1}$ be an IHR pmf. For all distribution $d_{2}, \frac{d_{1} * D_{2}}{D_{1}}(t)$ is a nondecreasing function of $t$.

Proof. The proof uses simple algebraic computations. Let us define the functions

$$
f_{u}(t) \stackrel{\text { def }}{=}\left\{\begin{array}{ll}
0 & \text { if } t \geq u \\
\frac{d_{1}(t-u)}{D_{1}(t)} D_{2}(u) & \text { if } 0<t<u
\end{array} .\right.
$$

With this definition we have $\frac{d_{1} * D_{2}}{D_{1}}(t)=\sum_{u=1}^{t-1} \frac{d_{1}(t-u)}{D_{1}(t)}=\sum_{u \in \mathbb{N}^{*}} f_{u}(t)$.

Let $h$ be the hazard rate of $d_{1}$, and $u$ be in $\mathbb{N}^{*}$. By definition of $h, \frac{d_{1}(t-u)}{D_{1}(t)}=$ $\frac{d_{1}(t-u)}{D_{1}(t-u)} \frac{D_{1}(t-u)}{D_{1}(t)}=h(t-u) \frac{1}{\prod_{v=1}^{u}(1-h(t-v))}$. Since the function $x \mapsto \frac{1}{1-x}$ is positive and increasing on $\left[0,1\left[\right.\right.$, if $h$ is non-decreasing, then $t \mapsto f_{u}(t)$ is non-decreasing as a product of positive and non-decreasing functions. Consequently, $t \mapsto \frac{d_{1} * D_{2}}{D_{1}}(t)$ is nondecreasing.

Monotony is a stronger but very interesting requirement: if the ratio is non-decreasing then the estimation never come back to state 1 after having decode state 2 . 


\section{Extension to $N$ states}

The previous arguments cannot be directly generalized to more than two states without further assumptions. Our next argument extends the idea of monotony that proposition 3 highlights. This approach turns out to be related to the notions of stochastic orderings introduced by [10].

Definition 3. Let $p_{1}, p_{2}$ be two distributions. $p_{1}$ is said to be locally smaller that $p_{2}$, denoted $p_{1} \leq p_{2}$, if $n \mapsto p_{1}(n) / p_{2}(n)$ is non-decreasing on $\operatorname{supp}\left[p_{2}\right]$.

A family $\left(p_{t}\right)_{t \in I}$ of pmfs indexed by $I \subset \mathbb{R}$ is said to be locally increasing if $\forall t_{1}, t_{2} \in$ $I, \quad t_{1} \leq t_{2} \Rightarrow p_{t_{1}} \leq p_{t_{2}}$.

Lemma 1. If $\left(p_{t}\right)_{t \in I}$ is an increasing family, then the mode $\mathbf{M}\left[p_{t}\right]$ of $p_{t}$ is a nondecreasing function of $t$.

This straightforward lemma is interesting: if the state probabilities of the semi-Markov chain $\left.(\mathbf{F}(t))_{t \in \mathbb{N}^{*}}\right)$ constitute an increasing family, then states are decoded with respect to their ordering - although some states might be skipped. Moreover, checking numerically the criterion on a given chain becomes very easy: it holds if and only if $\frac{F_{j+1}}{F_{j}}\left(l_{1}+\ldots+\right.$ $\left.l_{j}\right) \leq 1$ and $\frac{F_{j+1}}{F_{j}}\left(1+l_{1}+\ldots+l_{j}\right)>1$ for all $j$. So, to finish with, next proposition gives a sufficient condition to obtain an increasing process.

Definition 4. A discrete distribution $d$ is log-concave if for all $n$ in $\mathbb{N}, d(n)^{2} \geq d(n-$ 1) $d(n+1)$. This is equivalent to $d(.) \leq d(.+u)$ for all $u \in \mathbb{N}$.

It is noteworthy that all log-concave distributions are IHR. The main point is that log-concavity "preserve" stochastic ordering, as the following lemma explains.

Lemma 2 ([10, Theorem 2.1]). Let $f, g, h$ be three distributions. If $f$ is log-concave, then $g \underset{\text { lr }}{\leqslant} \quad \Rightarrow \quad f * g \underset{\mathrm{lr}}{\leqslant} * h$.

See the reference for its proof, that is an application of the Binet-Cauchy formula.

Proposition 4. If the semi-Markov chain is linear and all occupancy distributions $d_{j}$ are log-concave, then the process $(\mathbf{F}(t))_{t \in \mathbb{N}^{*}}$ is locally increasing.

Proof. Let $j$ be in $\mathbb{N}^{*}$. Since $d_{j-1}$ is log-concave, it is also IHR and $D_{j-1} \leqslant d_{j-1} * D_{j}$.

If $j>1$, let us consider $\frac{F_{j+1}}{F_{j}}=\frac{d_{1} * \ldots * d_{j-1} * d_{j} * D_{j+1}}{d_{1} * \ldots * d_{j-1} * D_{j}}$ The class of log-concave distributions is stable by convolution [10], so $d_{1} * \ldots * d_{j-1}$ is log-concave. Then, lemma 2 implies that $t \mapsto \frac{F_{j+1}(t)}{F_{j}(t)}$ is increasing.

The monotony of Markov processes has been largely studied - see [11] for a survey. Proposition 4 is a first step towards its extension to semi-Markov processes. Moreover, in locally increasing Markov chains, first-time passages $T_{j} \stackrel{\text { def }}{=} \inf \left\{t \mid X_{t+1} \geq j, X_{1}=1\right\}$ have log-concave pmfs [12]. Proposition 4 looks like a "reverse" counterpart of this result for linear semi-Markov chains, since the pmf of $T_{j+1}$ is $d_{1} * \ldots * d_{j}$ for such chains. 
As a conclusion, log-concavity seems to be the most desirable property for prior distributions of duration. While log-concavity plays an important role in many fields of statistics, it has been scarcely studied on HSMMs. It is highlighted by [13] for improving computational efficiency of the Viterbi algorithm. Proposition 4 shows it also provides theoretical coherency to the Forward estimation. Furthermore, experiments show that taking into account these prescriptions do improve the performances of realtime alignment. A comparative test with results and video files can be found on http: //repmus.ircam. fr/mutant/mlsp14.

\section{CONCLUSION \& PERSPECTIVES}

This paper introduces a criterion of time-coherent modeling in semi-Markov models for alignment. This criterion is about estimation coherency under non-discriminative observation. We show that coherency cannot be obtained with Viterbi estimation but can be obtained with is the Forward estimation if the chosen probability distributions have some precise properties. This short study calls for further theoretical and experimental developments. More necessary and sufficient conditions related to the criterion can be derived. The framework can be extended to other estimators such as the Forwardbackward algorithms. Moreover the proposed prescriptions lead to constraints on the learning parameter space; adding these constraints in HSMM training algorithms would be an interesting issue.

\section{REFERENCES}

1. K. P. Murphy, Dynamic Bayesian Networks: Representation, Inference and Learning, Ph.D. thesis, UC Berkeley, Computer Science Division (2002).

2. L. R. Rabiner, Proc. of the IEEE 77, 257-286 (1989).

3. A. Cont, IEEE Transaction on Pattern Analysis and Machine Intelligence 32, 974-987 (2010).

4. S. E. Levinson, Comput. Speech Lang. 1, 29-45 (1986), ISSN 0885-2308.

5. Y. Guédon, Journal of Computational and Graphical Statistics 12, 604-639 (2003), URL http: //hal.inria.fr/hal-00826992.

6. C. D. Mitchell, and L. H. Jamieson, "Modeling duration in a hidden Markov model with the exponential family," in Acoustics, Speech, and Signal Processing, 1993. ICASSP-93., 1993 IEEE International Conference on, 1993, vol. 2, pp. 331-334 vol.2, ISSN 1520-6149.

7. C. Joder, S. Essid, and G. Richard, "An Improved Hierarchical Approach for Music-to-symbolic Score Alignment," in ISMIR, edited by J. S. Downie, and R. C. Veltkamp, International Society for Music Information Retrieval, 2010, pp. 39-45, ISBN 978-90-393-53813.

8. S.-Z. Yu, and H. Kobayashi, Signal Process. 83, 235-250 (2003), ISSN 0165-1684, URL http: //dx.doi.org/10.1016/s0165-1684(02)00378-X.

9. S. Foss, and D. Korshunov, The Annals of Probability 35, 366-383 (2007), URL http://dx . doi.org/10.1214/009117906000000647.

10. J. Keilson, and U. Sumita, The Canadian Journal of Statistics / La Revue Canadienne de Statistique 10, pp. 181-198 (1982), ISSN 03195724.

11. M. Kijima, Journal of Applied Probability 35, pp. 545-556 (1998), ISSN 00219002, URL http: //www. jstor.org/stable/3215630.

12. S. Karlin, Total positivity (1968), URL http: / / opac. inria. fr/ record=b1089884.

13. D. Tweed, R. Fisher, J. Bins, and T. List, "Efficient Hidden Semi-Markov Model Inference for Structured Video Sequences," in Visual Surveillance and Performance Evaluation of Tracking and Surveillance, 2005. 2nd Joint IEEE International Workshop on, 2005, pp. 247-254. 